\title{
Indirect dark-matter searches with gamma-rays: Current and future gamma-ray observations from $\mathrm{KeV}$ to TeV
}

\author{
Aldo Morselli* \\ INFN Roma Tor Vergata, Italy \\ E-mail: aldo.morselli@roma2.infn.it
}

High-energy phenomena in the cosmos, and in particular processes leading to the emission of gamma- rays in the energy range $300 \mathrm{KeV}-100 \mathrm{TeV}$, play a very special role in the understanding of our Universe. This energy range is indeed associated with non-thermal phenomena and challenging particle acceleration processes. The Universe can be thought as a context where fundamental physics, relativistic processes, strong gravity regimes, and plasma instabilities can be explored in a way that is not possible to reproduce in our laboratories. High-energy astrophysics and atmospheric plasma physics are indeed not esoteric subjects, but are strongly linked with our daily life. Understanding cosmic high-energy processes has a large impact on our theories and laboratories applications. The technology involved in detecting gamma-rays is challenging and drives our ability to develop improved instruments for a large variety of applications.

The energy range between $300 \mathrm{Kev}$ and $100 \mathrm{MeV}$ is an experimentally very difficult range and remained uncovered since the time of COMPTEL. In this range a new instrument can address all astrophysics issues left open by the current generation of instruments. In particular better angular resolution in the energy range $10 \mathrm{MeV}-1 \mathrm{GeV}$ is crucial to resolve patchy and complex features of diffuse sources in the Galaxy and in the Galactic Centre as well as increasing the point source sensitivity. This instrument can address scientific topics of great interest to the community, with particular emphasis on multifrequency correlation studies involving radio, optical, IR, X-ray, soft gamma-ray and $\mathrm{TeV}$ emission.

Above $100 \mathrm{MeV}$, thanks to the launch of the Fermi-LAT satellite and to the advent of a new generation of imaging air Cherenkov telescopes (H.E.S.S., MAGIC, VERITAS), several thousand gamma-ray sources are known today revealing an unexpected ubiquity of particle acceleration processes in the Universe.

Major scientific challenges are still ahead, such as the identification of the nature of Dark Matter, the discovery and understanding of the sources of cosmic rays, or the comprehension of the particle acceleration processes that are at work in the various objects.

The identification of the nature of Dark Matter can be done with the detection of gamma rays and cosmic rays from the annihilation or decay of dark matter particles. This is a promising method for identifying dark matter, understanding its intrinsic properties, and mapping its distribution in the universe.

Dark Side of the Universe

15-18 Dec. 2015

Kyoto

${ }^{*}$ Speaker. 


\section{Search for Dark Matter in the Galactic Center and in the dwarf spheroidal galaxy satellites}

Astrophysical searches for dark matter (DM) are a fundamental part of the experimental efforts to explore the dark sector. The strategy is to search for DM annihilation products in preferred regions of the sky, i.e., those with the highest expected DM concentrations and still close enough to yield high DM-induced fluxes at the Earth. For that reason, the Galactic Center (GC), nearby dwarf spheroidal galaxy (dSphs) satellites of the Milky Way, as well as local galaxy clusters are thought to be among the most promising objects for DM searches. In particular, dSphs represent very attractive targets because they are highly DM-dominated systems and are expected to be free from any other astrophysical gamma-ray emitters that might contaminate any potential DM signal. Although the expected signal cannot be as large as that from the GC, dSphs may produce a larger signal-to-noise $(S / N)$ ratio. This fact allows us to place very competitive upper limits on the gamma-ray signal from DM annihilation [1, 2, 3], using data collected by the Large Area Telescope (LAT) onboard the Fermi gamma-ray observatory [4]. These are often referred to as the most stringent limits on DM annihilation cross-section obtained so far.

Despite these interesting limits derived from dSphs, the GC is still expected to be the brightest source of DM annihilations in the gamma-ray sky by several orders of magnitude. Although several astrophysical processes at work in the crowded GC region make it extremely difficult to disentangle the DM signal from conventional emissions, the DM-induced gamma-ray emission is expected to be so large there that the search is still worthwhile. Furthermore, the DM density in the GC may be larger than what is typically obtained in $N$-body cosmological simulations. Ordinary matter (baryons) dominates the central region of our Galaxy [5]. Thus, baryons may significantly affect the DM distribution. As baryons collapse and move to the center they increase the gravitational potential, which in turn forces the DM to contract and increase its density. This is a known and qualitatively well understood physical process $[6,8,9]$. It is also observed in many cosmological simulations that include hydrodynamics and star formation [10,11, 12, 13, 14, 15]. If this is the only effect of baryons, then the expected annihilation signal will substantially increase [5, 7].

A preliminary analysis of Fermi LAT observations of the GC region was presented in [20], [21], with an observation of an excess of gamma rays in the $3-5 \mathrm{GeV}$ energy range from the GC region. These results produced a lot of activity outside the Fermi collaboration with claims of evidence for dark matter in the Galactic Center (i.e. [22] [23] and references therein).

The possibility of a gamma-ray signal from DM annihilation from the GC was already considered in the analysis of the EGRET galactic center excess [35] but there are other possible explanations, e.g. a population of millisecond pulsars around the Galactic Center below the Fermi threshold [26], [27].

A third possibility is related to past activity of the Galactic Center [24],[25]. In this case the excess can be connected to the Fermi bubble and it will be very important to see how this bubble is structured in the GC region.

The analysis of the Fermi Collaboration [28] using 5 years of data and the Pass 7 event selections [29] and the on-going analysis with 6.5 year of data and the Pass 8 event selections [30] confirm the excess but confirm also that when all the uncertainties on the excess morphology and spectrum related to the modeling of the various components of gamma-ray emission in that region, 
in particular in the distribution of interstellar gas along the line of sight, in the low latitude emission from the Fermi bubbles and in the abundance of cosmic ray sources in the innermost Galaxy are considered, the spectrum varies significantly and it is not possible to discriminate between the different hypotesis.
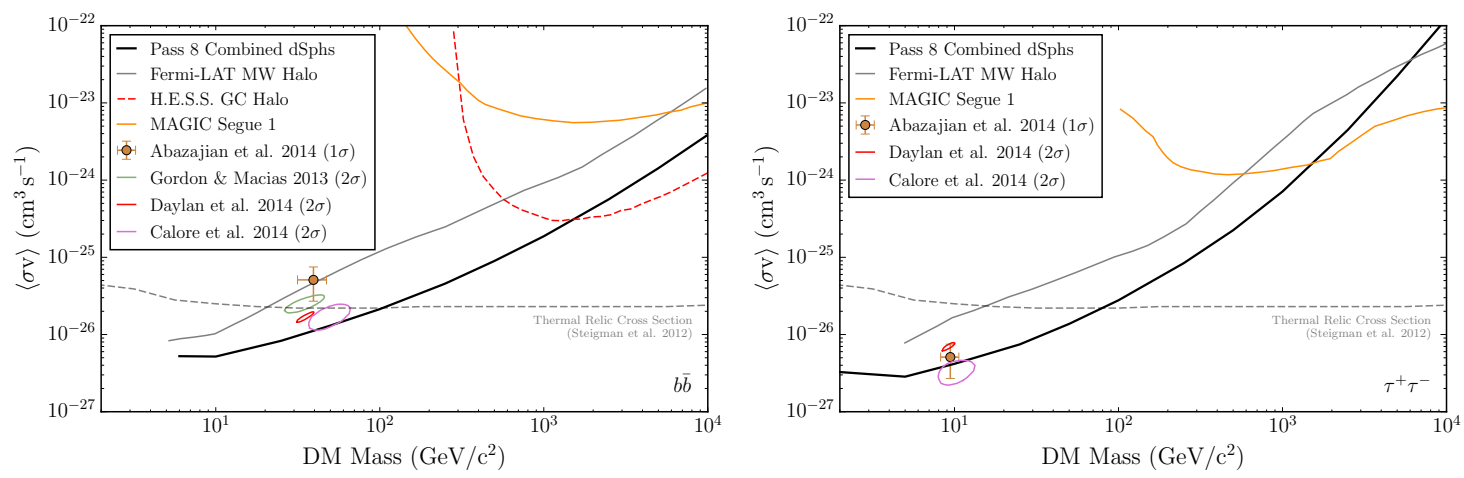

Figure 1: Comparison of constraints on the DM annihilation cross section for the $\bar{b} b(l e f t)$ and $\bar{\tau} \tau$ (right) channels [3] with previously published constraints from LAT analysis of the Milky Way halo ( $3 \sigma$ limit) [32], 112 hours of observations of the Galactic Center with H.E.S.S. [16], and 157.9 hours of observations of Segue 1 with MAGIC [17]. Closed contours and the marker with error bars show the best-fit cross section and mass from several interpretations of the Galactic center excess [22].

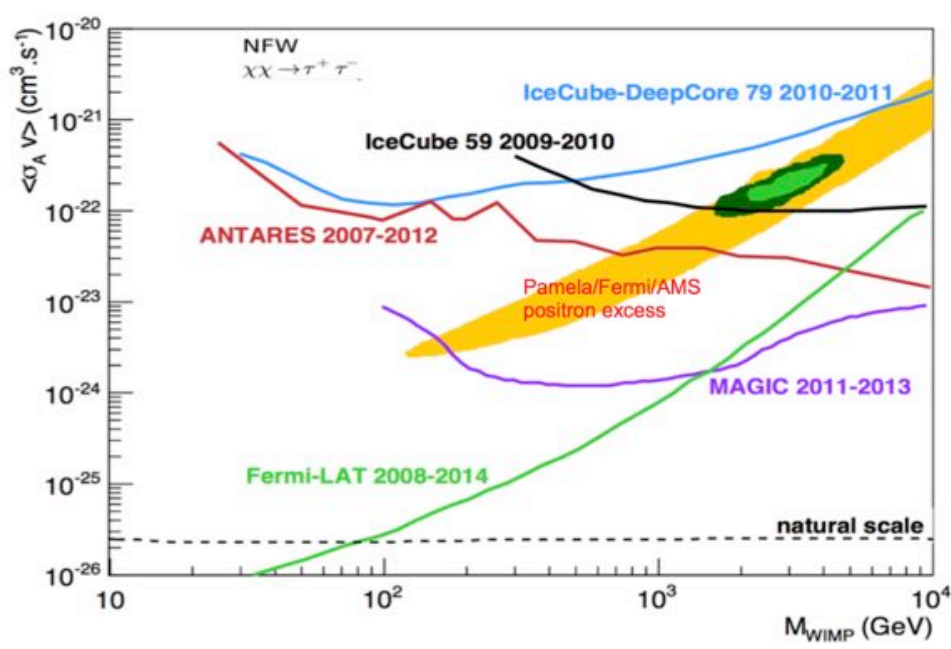

Figure 2: Comparison of constraints on the DM annihilation cross section for the $\bar{\tau} \tau$ channel [3] with Antares [18], IceCube-DeepCore [19] and MAGIC [17]

The new analysis of the dSphs with the use of Pass 8 begin to constrain some of the preferred parameter space for a DM interpretation of a gamma-ray excess in the Galactic center region.

As shown in 1 , for interpretations assuming a $\bar{b} b$ final state, the best-fit models lie in a region of parameter space slightly above the $95 \%$ CL upper limit from this analysis, with an annihilation cross section in the range of $(1-3) \times 10^{-26} \mathrm{~cm}^{3} \mathrm{~s}^{-1}$ and $m_{D M}$ between 25 and $50 \mathrm{GeV}$. However, 
uncertainties in the structure of the Galactic DM distribution can significantly enlarge the bestfit regions of $\langle\sigma v\rangle$ channel, and $\mathrm{m}_{D M}$. Figure 2 shows a comparison of constraints on the DM annihilation cross section for the $\bar{\tau}$ channel [3] with Antares [18], IceCube-DeepCore [19] and MAGIC [17]. One can see that the Fermi limits are the best limits below $2 \mathrm{TeV}$.

At lower energies a new instrument like Gamma-Light [36] , or ASTROGAM [37] can really improve these results both in the Galactic center and in the dSphs limits.

The project for an improved version of ASTROGAM, e-ASTROGAM, is being prepared in reply of the fifth ESA call for medium mission ( M5 ) that will be released in the fall of 2016 [38].

Of course this kind of mission will not only important for Dark Matter search but also for others fondamental searches as briefly described below;

\subsection{Physics and Cosmology with Gamma-Ray Bursts}

Since their serendipitous discovery more than four decades ago, most gamma-ray bursts (GRBs) have been shown to originate at cosmological distances, in the catastrophic death of massive stars. The collapse of a massive star into a black hole releases a huge amount of gravitational energy and surrounding material is accelerated to near the speed of light in a narrow jet, producing the luminous flash of gamma-rays that allows us to see these cosmic lighthouses across much of the observable universe. The nearest GRBs from such collapsars are excellent electromagnetic counterpart candidates to gravitational wave (GW) events. In addition, coalescing binaries, thought to be the progenitors of the short subset of GRBs, are widely viewed as the most promising sources of gravitational waves. The detection and localisation of these events is therefore a priority in the new era of gravitational wave astronomy and in the future era of space-based GW measurements. Because of their huge luminosities (up to $\geq 1053$ erg radiated in few tens of seconds) emitted by the most relativistic jets known and their redshift distribution extending up to at least $\mathrm{z} \sim 9$, GRBs offer enormous potential as powerful probes of the early Universe (evolution of stars, galaxies and the inter-galactic medium up to the epoch of re-ionization, popIII stars), as test-beds for fundamental physics (e.g., constraining limits on violations of Lorentz invariance), and as laboratories for matter and radiation under extreme conditions.

Sensitive measurements by e-ASTROGAM will allow a substantial step forward in these GRB-related research areas which are of extreme interest for several fields of astrophysics, cosmology and fundamental physics, and will provide an ideal synergy with the large multi-wavelength and multi-messenger faciltities that will be operative in the next decade (e.g., eLISA, E-ELT, SKA, CTA, ATHENA, neutrino observatories).

\subsection{Nuclear Sciences}

Gamma-ray line emission in the $\mathrm{MeV}$ domain is obtained through the decay/de-excitation of radioactive/stable nuclei which have been produced/excited by high-energy astrophysical phenomena, like supernova explosions or cosmic rays. They provide unique information on the isotopic identity of the emitters, on the underlying physical processes (e.g. nucleosynthesis, spallation etc.) and on the physical conditions of the otherwise inaccessible emitting region.

The $511 \mathrm{keV}$ emission from electron-positron annihilation in the Galaxy is the brightest gammaray line in the sky and the first ever detected from outside the solar system. Despite more than 40 
years of intense observational and theoretical investigation, the origin of annihilating positrons remains a mystery. The emission is strongly concentrated toward the Galactic bulge and the bulge/disk ratio appears higher than observed in any other wavelength. From the theoretical point of view it is unclear what are the main sources of positrons: conventional (like gravitational and thermonuclear supernovae, or Low-mass X-ray binaries and microquasars), or more exotic (like de-exciting dark matter particles or the Galactic supermassive black hole which, however, is notoriously inactive today). Furthermore, positrons may propagate for several $10^{5} \mathrm{yr}$ far away from their sources before annihilating, depending on still poorly understood properties of Galactic ISM and magnetic fields, making it difficult to infer positron sources from the observed gamma-ray emission. For those reasons, understanding the Galactic $511 \mathrm{KeV}$ emission constitutes a real challenge for modern astrophysics.

\subsection{Low energy cosmic-rays}

The question of the origin of cosmic-rays has been the subject of hot debates for decades now. The common view is that the kinetic energy of supernova explosions is transferred to relativistic particles during the propagation of the shock wave in the surrounding matter. Yet, many questions are left unanswered, since no direct unambiguous measurement exists so far. The role of cosmic rays in the physics and chemistry of the interstellar medium is also poorly known in the absence of any measurement of the lower part of their spectrum because of the solar magnetic field. Cosmic rays of energies below about $1 \mathrm{GeV} /$ nucleon are thought to play a key role in the chemistry and dynamics of the interstellar medium. First, low-energy cosmic rays are a primary source of ionization in shielded molecular cloud regions. The resulting ionization fraction drives the chemistry in these regions and controls the coupling of the gas with the ambient magnetic field, which in turns determines the dynamics of star formation in dense molecular cores. Cosmic rays also represent an important source of heating for molecular clouds, as the energy of secondary electrons produced by the ionization process is in large part converted into heat by inelastic collisions with ambient atoms and molecules. Observations of the molecule $\mathrm{H} 3+$ in diffuse clouds indicate an average cosmic-ray ionization rate of molecular hydrogen of about $410^{-16} s^{-1}$, which is more than an order of magnitude larger than the so-called standard rate. These observations imply that the average flux of low-energy cosmic rays in the Galaxy may be much higher than previously thought. Since the solar modulation effect makes impossible a direct detection of sub-GeV cosmic rays near Earth, the nuclear gamma-ray line emission produced by interaction of low-energy cosmic rays with interstellar nuclei represents the best chance to get information about the flux.

\section{The TeV range}

The Cherenkov Telescope Array (CTA) is the next generation gamma-ray observatory with a factor 10 better sensitivity compared to existing facilities and it has a unique chance of discovery of dark matter in the form of WIMPs.

The observational strategy proposed for the CTA Dark Matter programme is focused first on collecting a significant amount of data on the centre of the Galactic Halo. Complementary observations of a dSph galaxy will be conducted to extend the dark matter searches. The Galactic Halo and Large Magellanic Cloud are valuable targets both for dark matter searches and studies of 


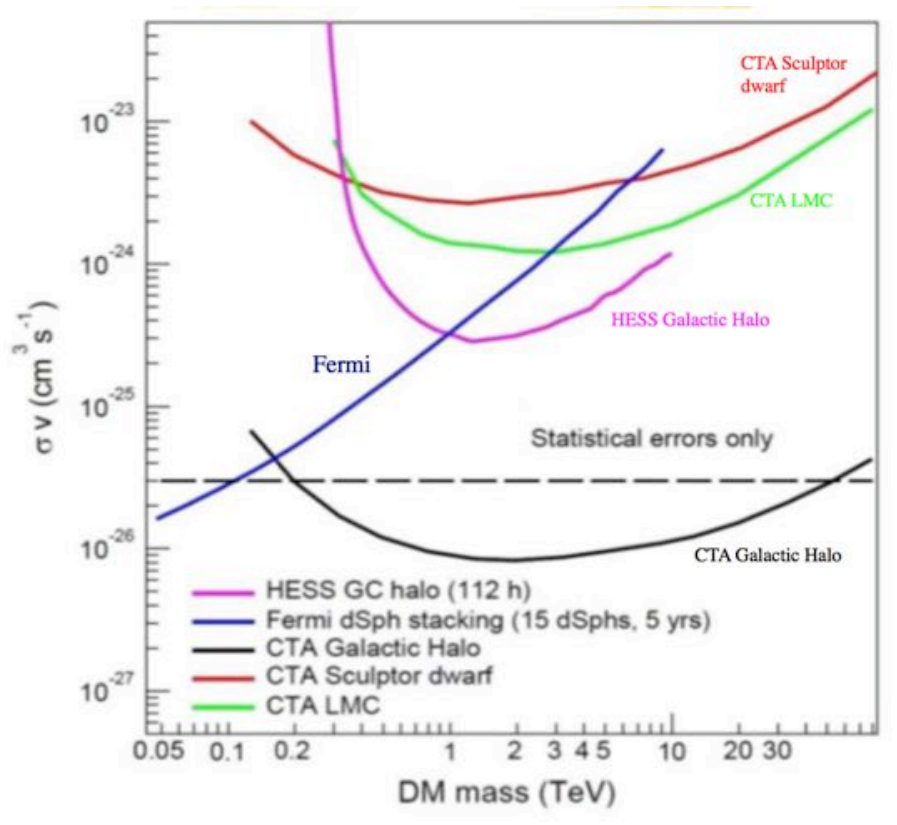

Figure 3: Comparison of predicted sensitivities for the targets of: the Milky Way Halo; the Large Magellanic Cloud and the dwarf galaxy Sculptor. The CTA sensitivity curves use the same method for each target and the NFW dark matter profile. The sensitivity calculations have a $30 \mathrm{GeV}$ threshold for the MW and Sculptor and $200 \mathrm{GeV}$ for the LMC. The sensitivities for the three targets are all for 500 hours taking into account only statistics errors; for the MW and the LMC, the systematics of backgrounds must be very well controlled to achieve this statistically possible sensitivity.

non-thermal processes in astrophysical sources. Data will be searched for continuum emission and line features, and strategies will be adopted according to findings. Clearly discoveries will modify any strategies defined a priori.

The inconvenience of Galactic Halo is the fact that being a diffuse source, the integration over the inner halo, while yielding a large signal, gives a very large instrumental background from misidentified charged cosmic rays. Further, there are astrophysical backgrounds from various sources which must be understood, even with the very central region excluded from the analysis. It is believed that the disadvantages of the MW target can be overcome with sufficient experimental effort to control systematic effects in background subtraction or modelling. VHE standard astrophysical processes have steeper spectra than the expected DM-induced gamma-ray continuum emission. Given the wealth of other high energy emitters expected in this region, the search for a DM component requires a very deep exposure to enable detection and detailed spectromorphological studies; a deep understanding of the instrumental and observational systematics; and accurate measurements of other astrophysical emission in the region to be able to reduce at best contamination to the DM signal. A deep exposure for the Galactic Centre observation will provide 


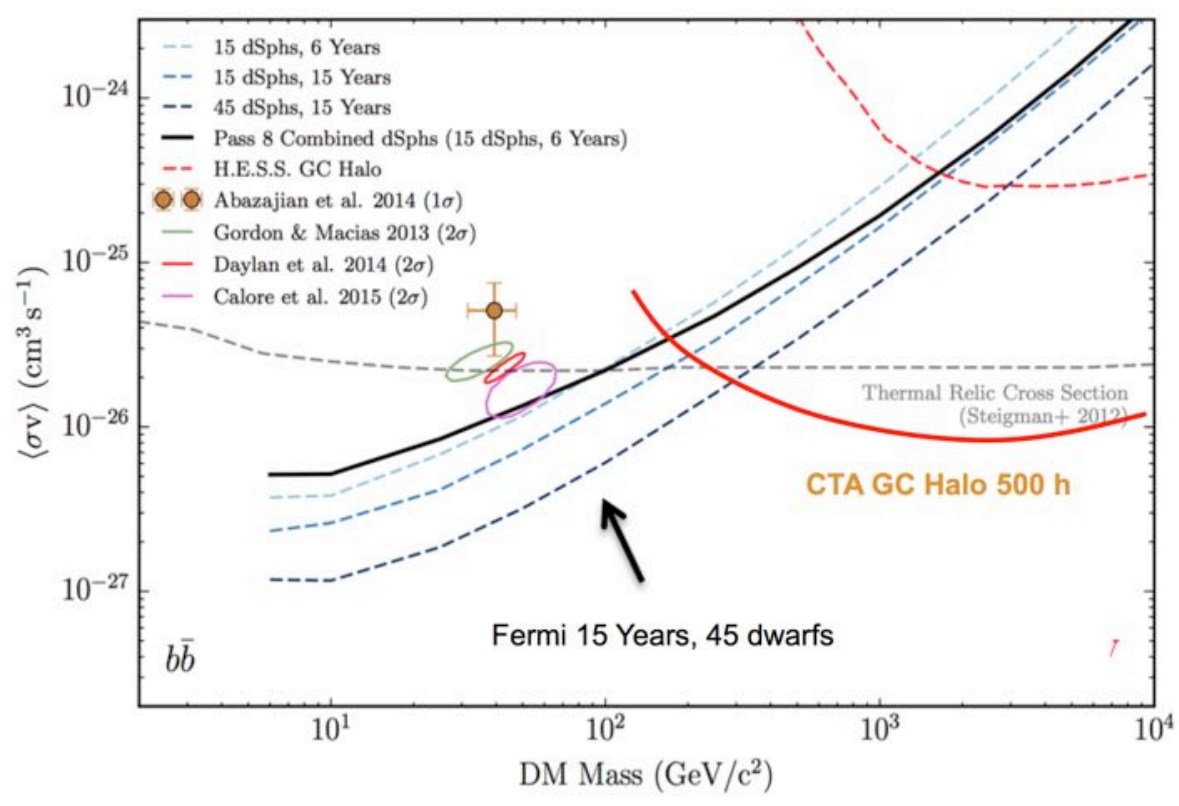

Figure 4: Projected upper limits on the WIMP annihilation cross section from the joint analysis of dSphs as a function of the size of the dSph sample on the assumption of 15 years and 45 dwarfts with Fermi together with the CTA halo upper limit.

the means for an in-depth study and better understanding of the astrophysical emissions in this region.

The sensitivity predictions for the Galactic Halo, the dwarf galaxy Sculptor and the Large Magellanic Cloud are compared in Figure 3. Here it can be clearly seen that the sensitivity possible with the Galactic Halo observation is much better than that which is possible with a single dwarf galaxy or the LMC. Although this plot does not show the effect of systematics, the relative rankings of the sensitivities is not changed by including them [39].

Figure 4 show the projected upper limits on the WIMP annihilation cross section from the joint analysis of dSphs as a function of the size of the dSph sample on the assumption of 15 years and 45 dwarfts with Fermi together with the CTA halo upper limit. Together Fermi and CTA will probe most of the space of WIMP models with thermal relic annihilation cross section.

To probe the region below $5 \mathrm{GeV}$ again we need an experiment like e-ASTROGAM.

\section{Conclusions}

Detection of gamma rays from the annihilation or decay of dark matter particles is a promising method for identifying dark matter, understanding its intrinsic properties, and mapping its distribution in the universe (in synergy with the experiments at the LHC and in the underground laboratories). In the future it would be extremely important to extend the energy range of experiments at lower energies (compared to the Fermi energies) ( ASTROGAM ) and higher energies (HAWC, CTA, LHAASO) 


\section{References}

[1] M. Ackermann et al. [Fermi-LAT Collaboration], Phys. Rev. Lett. 107 (2011) 241302 [arXiv:1108.3546 [astro-ph.HE]].

[2] A. A. Abdo et al. [Fermi-LAT Collaboration], Astrophys. J. 712 (2010) 147 [arXiv:1001.4531 [astro-ph.CO]].

[3] M. Ackermann et al. [Fermi LAT Collaboration], Phys. Rev. Lett. 115, 2313018 pg. (2015) [arXiv:1503.02641]

[4] W. B. Atwood et al. [Fermi-LAT Collaboration], Astrophys. J. 697 (2009) 1071 [arXiv:0902.1089 [astro-ph.IM]].

[5] F. Prada, A. Klypin, J. Flix Molina, M. Martinez and E. Simonneau, Phys. Rev. Lett. 93 (2004) 241301 [astro-ph/0401512].

[6] Ya. B. Zeldovich, A. A. Klypin, M. Yu. Khlopov and V. M. Chechetkin, Sov. J. Nucl. Phys. 31 (1980) 664.

[7] Y. Mambrini, C. Muñoz, E. Nezri and F. Prada, JCAP 01 (2006) 010 [hep-ph/0506204].

[8] G.R. Blumenthal, S.M. Faber, R. Flores and J.R. Primack, Astrophys. J. 301 (1986) 27.

[9] O. Y. Gnedin, A. V. Kravtsov, A. A. Klypin and D. Nagai, Astrophys. J. 616 (2004) 16 [astro-ph/0406247 [astro-ph]].

[10] M. Gustafsson, M. Fairbairn and J. Sommer-Larsen, Phys. Rev. D 74 (2006) 123522 [astro-ph/0608634 [astro-ph]].

[11] P. Colín, O. Valenzuela and A. A. Klypin, Astrophys. J. 644 (2006) 687 [astro-ph/0506627 [astro-ph]].

[12] P. B. Tissera, S. D. M. White, S. Pedrosa and C. Scannapieco, MNRAS 406 (2010) 922 [arXiv:0911.2316 [astro-ph.CO]].

[13] O. Y. Gnedin, D. Ceverino, N. Y. Gnedin, A. A. Klypin, A. V. Kravtsov, R. Levine, D. Nagai and G. Yepes, arXiv:1108.5736 [astro-ph.CO].

[14] M. Zemp, O. Y. Gnedin, N. Y. Gnedin and A. V. Kravtsov, Astrophys. J. 748 (2012) 54 [arXiv:1108.5384 [astro-ph.GA]].

[15] J. Sommer-Larsen and M. Limousin, MNRAS 408 (2010) 1998 [arXiv:0906.0573 [astro-ph.CO]].

[16] A. Abramowski et al. (H.E.S.S. Collaboration), Phys. Rev. Lett. 106, 161301 (2011) [arXiv:1103.3266]

[17] J. Aleksić et al. (MAGIC Collaboration), J. Cosmol. Astropart. Phys. 1402, 008 (2014) [arXiv:1312.1535]

[18] S. Adrían-Martínez et al. [Antares Coll.] arXiv:1505.04866

[19] M.G. Aartsen et al. [IceCube Collaboration] arXiv:1309.7007

[20] V. Vitale and A. Morselli for the Fermi LAT Collaboration, 2009 Fermi Symposium, eConf Proceedings C091122 [arXiv:0912.3828]

[21] A. Morselli, B.Cañadas, V.Vitale, Il Nuovo Cimento 34 C, N. 3 (2011) [arXiv:1012.2292]

[22] F.Calore et al. arXiv:1409.0042

[23] T. Daylan et al., 2014, arXiv:1402.6703 
[24] J. Petrovic et al., 2014, JCAP, 10, 052

[25] E.Carlson, S.Profumo , 2014, Phys. Rev. D, 90, 023015

[26] Lee et al. Phys. Rev. Lett. 116, 051103 (2016) [arXiv:1506.05124]

[27] R. Bartels et al. Phys. Rev. Lett. 116, 051102 (2016) [arXiv:1506.05104]

[28] M.Ajello et al. [Fermi-LAT Collaboration] The Astrophysical Journal 819:44 (30pp) 2016 [arXiv:1511.02938]

[29] M.Ackermann et al. [Fermi-LAT Collaboration] The Astrophysical Journal Supplement 203 (2012) 4, [arXiv:1206.1896]

[30] M. Ackermann et al. [Fermi LAT Collaboration], Phys. Rev. D. 91, 122002 (2015) [arXiv:1506.00013]

[31] G.A.Gomez-Vargas et al. JCAP 10 (2013) 029 [arXiv:1308.3515]

[32] M. Ackermann et al. [Fermi LAT Collaboration], Astrophys. J. 761 (2012) 91 [arXiv:1205.6474 [astro-ph.CO]].

[33] A. Birkedal, K. T. Matchev, M. Perelstein and A. Spray, hep-ph/0507194.

[34] G. Jungman, M. Kamionkowski and K. Griest, Phys. Rep. 267 (1996) 195 [arXiv:hep-ph/9506380].

[35] A.Cesarini, F.Fucito, A.Lionetto, A.Morselli, P. Ullio, Astropart. Phys. 21 (2004) 267 [astro-ph/0305075]

[36] A.Morselli et al., Nuclear Physics B - $239 Đ 240$ (2013) 193-198 [arXiv:1406.1071]

[37] http://astrogam.iaps.inaf.it

[38] C.Pittori, A.Morselli, DSU15 Proceedings

[39] J.Carr et al., POS(ICRC2015) 1203 arXiv:1508.06128 